\title{
Chemical shift magnetic resonance imaging in differentiation of benign from malignant vertebral collapse in a rural tertiary care hospital in North India
}

\author{
Puneet Mittal, Ranjana Gupta, Amit Mittal, Sandeep Joshi ${ }^{1}$ \\ Departments of Radiodiagnosis and ${ }^{1}$ Medicine, Maharishi Markandeshwar Institute of Medical Sciences and Research, Mullana, Ambala, \\ Haryana, India
}

\section{ABSTRACT}

Introduction: Magnetic resonance imaging (MRI) is the modality of the first choice for evaluation of vertebral compression/collapse. Many MRI qualitative features help to differentiate benign from malignant collapse. We conducted this study to look for a quantitative difference in chemical shift values in benign and malignant collapse using dual-echo gradient echo in-phase/out-phase imaging. Materials and Methods: MRI examinations of a total of 38 patients were retrospectively included in the study who had vertebral compression/collapse with marrow edema in which final diagnosis was available at the time of imaging/follow-up. Signal intensity value in the region of abnormal marrow signal and adjacent normal vertebra was measured on in phase/out phase images. Signal intensity ratio (SIR) was measured by dividing signal intensity value on opposite phase images to that on in phase images. SIR was compared in normal vertebrae and benign and malignant vertebral collapse. Results: There were 21 males and 17 females with mean age of 52.4 years (range $28-76$ years). Out of total 38 patients, 18 were of benign vertebral collapse and 20 of malignant vertebral collapse. SIR in normal vertebrae was $0.30 \pm 0.14,0.67 \pm 0.18$ in benign vertebral collapse, and $1.20 \pm 0.27$ in malignant vertebral collapse with significant difference in SIR of normal vertebrae versus benign collapse $(P<0.01)$ and in benign collapse versus malignant collapse $(P<0.01)$. Assuming a cutoff of $<0.95$ for benign collapse and $\geq 0.95$ for malignant collapse, chemical shift imaging had a sensitivity of $90 \%$ and specificity of 94.4\%. Conclusion: Chemical shift imaging is a rapid and useful sequence in differentiating benign from malignant vertebral collapse with good specificity and sensitivity.

Key words: Chemical, in-phase, magnetic resonance imaging, out-phase, shift, spine, vertebral collapse

\section{Introduction}

Acute vertebral collapse due to any cause is associated with marrow signal changes. Magnetic resonance imaging (MRI) is important in differentiating benign from malignant causes of vertebral collapse. Various morphological MR features have been used, but they

\section{Address for correspondence:}

Dr. Puneet Mittal, 448, Prem Basti, Sangrur (Pb.) - 148 001,

Punjab, India.

E-mail: drpuneetmittal@gmail.com

\begin{tabular}{|l|l|}
\hline \multicolumn{2}{|c|}{ Access this article online } \\
\hline Quick Response Code: & Website: \\
\hline & www.ruralneuropractice.com \\
\cline { 2 - 2 } & \\
\hline
\end{tabular}

lack specificity. Chemical shift imaging with in-phase/out phase imaging relies on differentiating tissues based on the relative proportion of fat and fluid. In malignant lesions, there is complete replacement of marrow fat by malignant cells, therefore in out-phase images, there should be no signal drop. Therefore, signal intensity ratio (SIR) of out-phase/in-phase images should be high. On the other hand, in benign lesions, there is incomplete marrow replacement; therefore, there should be signal drop on out-phase images, and SIR should be low. ${ }^{[1,2]}$ There are not many studies in Indian literature on the

This is an open access article distributed under the terms of the Creative Commons Attribution-NonCommercial-ShareAlike 3.0 License, which allows others to remix, tweak, and build upon the work non-commercially, as long as the author is credited and the new creations are licensed under the identical terms.

For reprints contact: reprints@medknow.com

How to cite this article: Mittal P, Gupta R, Mittal A, Joshi S. Chemical shift magnetic resonance imaging in differentiation of benign from malignant vertebral collapse in a rural tertiary care hospital in North India. J Neurosci Rural Pract 2016;7:489-92. 
usefulness of this technique. Therefore, in the present study, we compared SIR values in benign and malignant vertebral collapse with that of normal vertebrae.

\section{Materials and Methods}

The present study was conducted in Radiodiagnosis Department of Maharishi Markandeshwar Institute of Medial Sciences and Research, Mullana, Ambala, Haryana. Chemical shift imaging using dual-echo gradient echo sequence is routinely used in our MRI protocol for evaluation of vertebral collapse with associated marrow changes. The study was approved by the ethical committee. MRI examinations of a total of 38 patients from October 2014 to October 2015 were retrospectively included in the study who had vertebral compression/ collapse with marrow edema in which final diagnosis was available at the time of imaging/follow-up. Patients with previous spinal operative history, history of radiotherapy, and diffuse marrow signal abnormality were excluded from the study.

\section{Magnetic resonance imaging protocol}

All examinations were performed on a 16 channel 1.5 Tesla MRI scanner (Achieva, Philips Medical Systems, The Netherlands). Localizer was taken in axial and sagittal planes after making proper positioning of the patient. The MRI protocol consisted of sagittal T1-weighted (T1W), sagittal T2W, axial T1W, axial T2W sequences, and coronal short tau inversion recovery images. Sagittal in-phase/out-phase images were obtained using dual-echo gradient echo sequence with following parameters: TR-Minimum, TE1 - $2.4 \mathrm{~ms}$ (for out-phase images), TE2 - $4.6 \mathrm{~ms}$ (for in-phase images), flip angle- $90^{\circ}$, slice thickness $-5 \mathrm{~mm}$, acquisition time - approximately $20 \mathrm{~s}$.

\section{Magnetic resonance imaging evaluation}

Conventional MR sequences were evaluated for locating the vertebral compression/collapse and areas of marrow signal abnormality. The area of abnormal marrow signal was identified on in phase/out phase images. An elliptical/rounded region of interest (ROI) was placed at identical sites on in-phase/out-phase images using electronic calipers, and signal intensity was measured. SIR was measured by dividing signal intensity value on opposite-phase images to that on in-phase images. Signal intensity was also measured in adjacent vertebrae with normal marrow signal on both in phase/out phase images with the calculation of SIR of normal vertebrae. In cases with multifocal lesions with involvement of multiple vertebrae, only one abnormal vertebra was sampled.

\section{Results}

There were 21 males and 17 females with mean age of 52.4 years (range 28-76 years). Out of total 38 patients, 18 were of benign vertebral collapse. Benign vertebral collapse causes included: Osteoporotic (N-10), infective (N-6), and traumatic (N-2). Malignant vertebral collapse (twenty patients) causes included: Metastasis (N-16) and multiple myeloma/plasmacytoma $(\mathrm{N}-4)$. Based on the analysis of MR sequences and calculation of SIRs, the values in normal vertebrae, benign, and malignant vertebrae are listed in Table 1. SIR in normal vertebrae was $0.30 \pm 0.14$. In benign vertebral collapse, it was $0.67 \pm 0.18$ [Figures 1 and 2] and in malignant vertebral collapse, it was $1.20 \pm 0.27$ [Figure 3]. There was significant difference in SIR of normal vertebrae versus benign collapse $(P<0.01)$ and in benign collapse versus malignant collapse $(P<0.01)$. Assuming a cutoff of $<0.95$ for benign collapse and $\geq 0.95$ for malignant collapse, chemical shift imaging was able to correctly identify 17 out of 18 benign vertebral collapse and 18 out of 20 malignant vertebral collapse (Sensitivity-90\%, specificity $-94.4 \%$, positive predictive value $-94.7 \%$, and negative predictive value $-89.4 \%$ ).

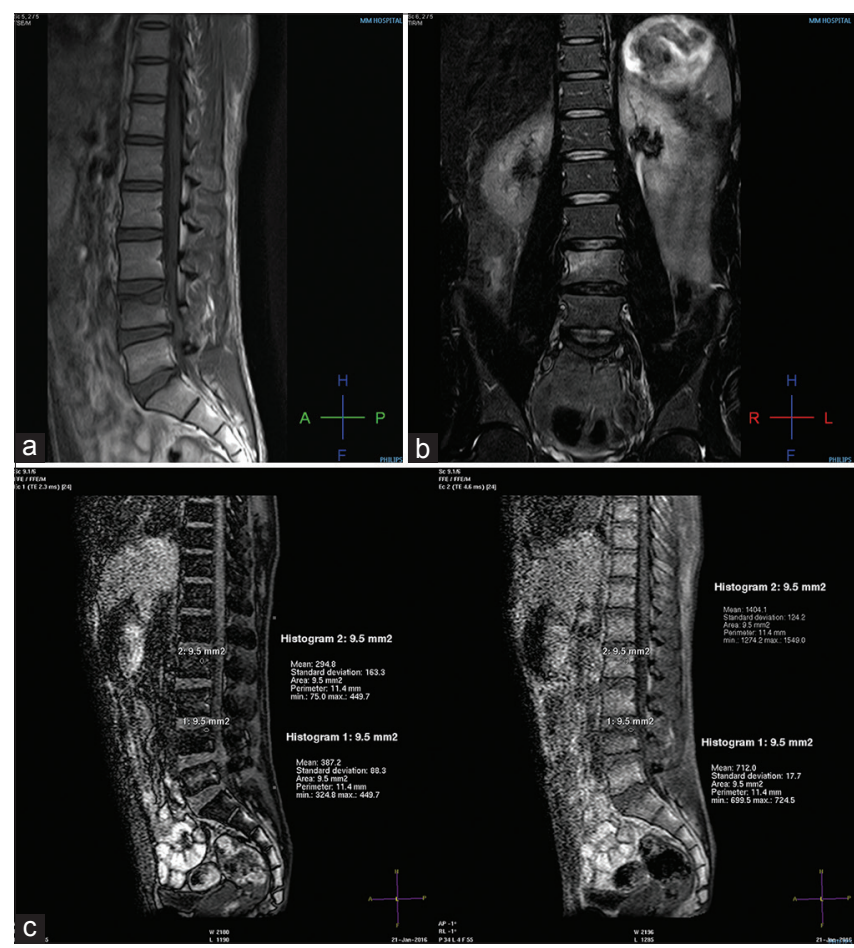

Figure 1: Sagittal T1-weighted (a) and coronal short tau inversion recovery (STIR) images (b) showing mild anterior wedging of L4 vertebra with marrow signal abnormality along its superior end plate. Sagittal in-phase/out-phase images (c) showing signal drop on out-phase images with signal intensity ratio of 0.54 consistent with benign vertebral collapse 


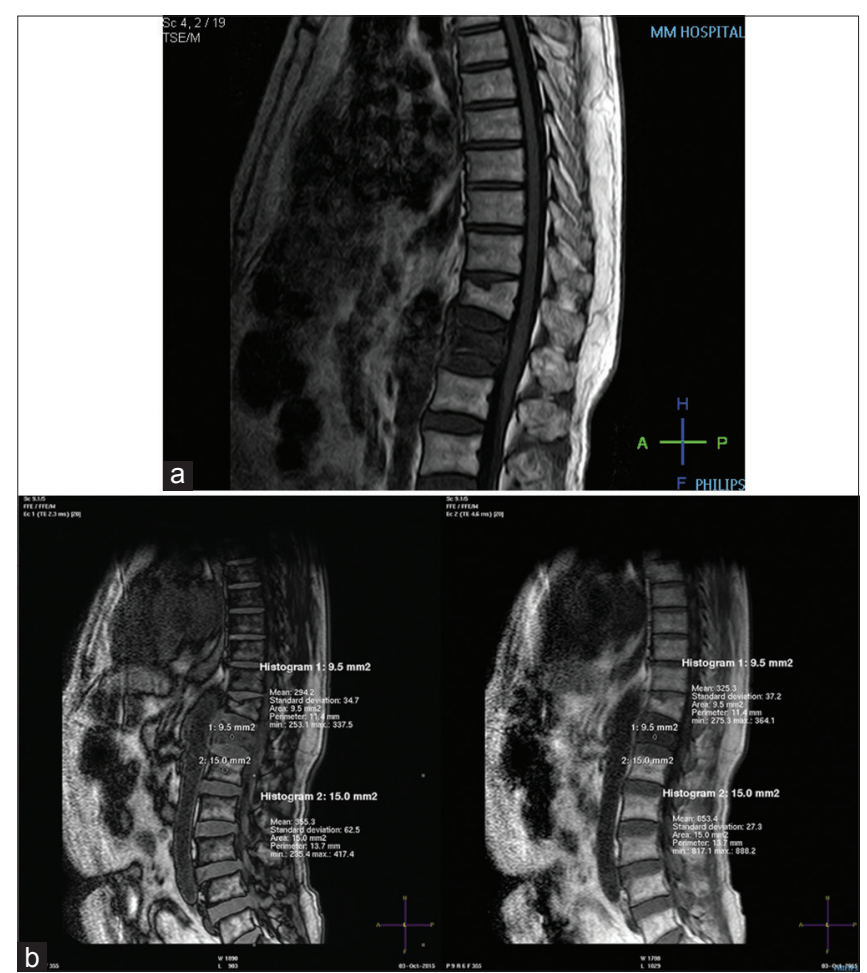

Figure 2: Sagittal T1-weighted image (a) showing partial collapse of D12 vertebra with marrow signal abnormality. Sagittal in-phase/out-phase images (b) showing partial signal drop on out-phase images with signal intensity ratio of 0.90 consistent with benign collapse

\section{Table 1: Showing signal intensity ratios (out-phase/ in-phase)}

\begin{tabular}{lc}
\hline & Signal intensity ratio (Mean \pm SD) \\
\hline Normal vertebrae (38) & $0.30 \pm 0.14$ \\
Benign (18) & $0.67 \pm 0.18$ \\
Malignant (20) & $1.20 \pm 0.27$ \\
\hline
\end{tabular}

\section{Discussion}

Chemical shift imaging is based on the principle that precession frequencies of hydrogen protons in water and fat molecules are slightly different, which means that after excitation; they will repeatedly be in-phase and out-phase. When in-phase, the signal of fat and water protons will be added. When out-phase (water and fat protons at $180^{\circ}$ to each other), the signal of fat and water protons will nullify each other. Therefore, in voxels containing both water and fat molecules, there will be drop of the signal in out-phase images.

Rapid dual-echo gradient echo imaging allows fast acquisition at precise echo times to produce in-phase and out-phase images in a single sequence. At 1.5 Tesla systems, out-phase images are typically acquired at $2.4 \mathrm{~ms}$ and in-phase images at $4.6 \mathrm{~ms}^{\left[{ }^{[1]}\right.}$ Due to very rapid and simultaneous acquisition, both in-phase and out-phase images are identical which allows precise

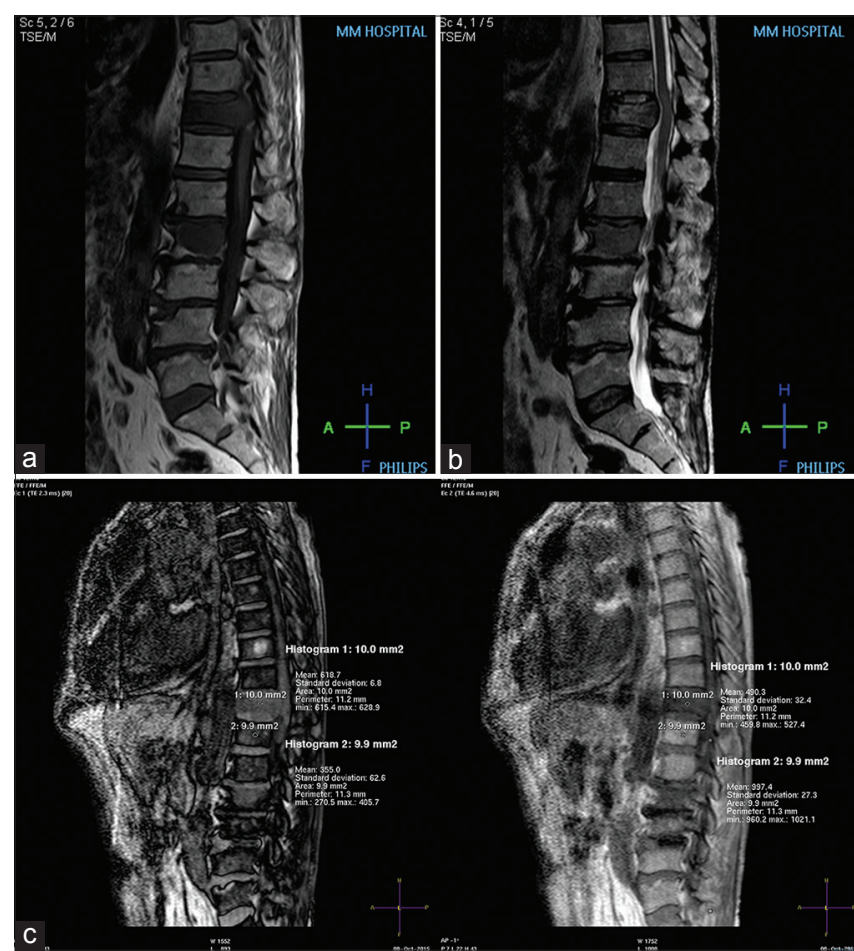

Figure 3: Sagittal T1-weighted (a) and T2-weighted images (b) showing partial collapse of D11 vertebra with marrow signal abnormality in D11 and L2 vertebrae. Sagittal in-phase/out-phase images (c) showing no signal drop on out-phase images with signal intensity ratio of 1.26 consistent with malignant collapse

placement of ROI at identical sites in both images. Due to rapid acquisition within a matter of seconds, it adds very little to the imaging time which is an important consideration when imaging old/debilitated patients.

Normal vertebrae contain roughly equal amount of fat and water protons. Therefore, they show loss of signal on out-phase images with low SIR. In benign vertebral collapse, there is partial replacement of fat with fluid with resultant increase in SIR as compared to normal vertebrae. However, since the replacement is partial, there is still signal drop on out-phase images. In malignant marrow diseases, there is almost complete marrow replacement with tumor cells resulting in highest SIR values. ${ }^{[1,3,4]}$

In this study, there was significant difference in SIR of normal vertebra versus benign vertebral collapse and benign versus malignant vertebral collapse $(P<0.01)$. These results are similar to previous studies. ${ }^{[1,2,5,6]}$

In this study, using a cutoff of $<0.95$ for benign collapse and $\geq 0.95$ for malignant collapse, we were able to correctly identify 17 out of 18 benign vertebral collapse and 18 out of 20 malignant vertebral collapse with sensitivity of $90 \%$ and specificity of $94.4 \%$. Other authors have defined cutoff values of $0.8-0.91$ with similar 
sensitivity and specificity. ${ }^{[1,2]}$ Due to the fact that there is some variability in the degree of marrow replacement in both benign and malignant processes, there will always be some false positive and negative results based on chemical shift imaging, regardless of what cut-off has been used. However, its high specificity is helpful to reduce errors. Moreover, chemical shift imaging, when viewed along with other MR findings can help to reach the correct diagnosis.

\section{Conclusion}

Chemical shift imaging with in-phase/out-phase imaging is a robust and rapid sequence which can provide useful information in differentiating benign from malignant vertebral collapse. Therefore, it should be included in routine MRI for evaluation of vertebral collapse.

\section{Financial support and sponsorship}

Nil.

\section{Conflicts of interest}

There are no conflicts of interest.

\section{References}

1. Erly WK, Oh ES, Outwater EK. The utility of in-phase/opposed-phase imaging in differentiating malignancy from acute benign compression fractures of the spine. AJNR Am J Neuroradiol 2006;27:1183-8.

2. Zidan DZ, Habib LA, Chalabi NA. Quantitative chemical-shift MR imaging cutoff value: Benign versus malignant vertebral compression - Initial experience. Egypt J Radiol Nucl Med 2014;45:779-86.

3. Gokalp G, Mutlu FS, Yazici Z, Yildirim N. Evaluation of vertebral bone marrow fat content by chemical-shift MRI in osteoporosis. Skeletal Radiol 2011;40:577-85.

4. Ishijima H, Ishizaka H, Horikoshi H, Sakurai M. Water fraction of lumbar vertebral bone marrow estimated from chemical shift misregistration on MR imaging: Normal variations with age and sex. AJR Am J Roentgenol 1996;167:355-8.

5. Disler DG, McCauley TR, Ratner LM, Kesack CD, Cooper JA. In-phase and out-of-phase MR imaging of bone marrow: Prediction of neoplasia based on the detection of coexistent fat and water. AJR Am J Roentgenol 1997;169:1439-47.

6. $\quad$ Eito K, Waka S, Naoko N, Makoto A, Atsuko H. Vertebral neoplastic compression fractures: Assessment by dual-phase chemical shift imaging. J Magn Reson Imaging 2004;20:1020-4.

\section{Author Help: Reference checking facility}

The manuscript system (www.journalonweb.com) allows the authors to check and verify the accuracy and style of references. The tool checks the references with PubMed as per a predefined style. Authors are encouraged to use this facility, before submitting articles to the journal.

- The style as well as bibliographic elements should be $100 \%$ accurate, to help get the references verified from the system. Even a single spelling error or addition of issue number/month of publication will lead to an error when verifying the reference.

- Example of a correct style

Sheahan P, O'leary G, Lee G, Fitzgibbon J. Cystic cervical metastases: Incidence and diagnosis using fine needle aspiration biopsy. Otolaryngol Head Neck Surg 2002;127:294-8.

- Only the references from journals indexed in PubMed will be checked.

- $\quad$ Enter each reference in new line, without a serial number.

- Add up to a maximum of 15 references at a time.

- If the reference is correct for its bibliographic elements and punctuations, it will be shown as CORRECT and a link to the correct article in PubMed will be given.

- If any of the bibliographic elements are missing, incorrect or extra (such as issue number), it will be shown as INCORRECT and link to possible articles in PubMed will be given. 